\title{
Volumetric laser endomicroscopy can target neoplasia not detected by conventional endoscopic measures in long segment Barrett's esophagus
}

Authors

Institution
Arvind J. Trindade, Benley J. George, Joshua Berkowitz, Divyesh V. Sejpal, Matthew J. McKinley

Hofstra North Shore-Long Island Jewish School of Medicine, North Shore University Hospital, Manhasset, NY, United States submitted

25. August 2016

accepted after revision

4. January 2016

\section{Bibliography}

DOI http://dx.doi.org/

10.1055/s-0042-101409

Endoscopy International Open 2016; 04: E318-E322

(c) Georg Thieme Verlag KG

Stuttgart · New York

E-ISSN 2196-9736

\section{Corresponding author}

Arvind J. Trindade

North Shore University Hospital

Division of Gastroenterology

300 Community Drive

Manhasset, NY 11030

Fax: +1-718-343-0128

arvind.trindade@gmail.com
License terms

(이 (1) $\ominus \circledast$
Methods and study aims: The incidence of esophageal cancer is rising despite increased surveillance efforts. Volumetric laser endomicroscopy (VLE) is a new endoscopic imaging tool that can allow for targeted biopsy of neoplasia in Barrett's esophagus. We report a series of 6 patients with long-segment Barrett's esophagus ( $>3 \mathrm{~cm}$ ), who underwent a session of endoscopy with volumetric laser endomicroscopy, after a separate prior session of standard high-definition endoscopy with narrow band imaging (NBI) and random biopsies that did not reveal neoplasia. In all six patients, the first endoscopy was the index endos-

\section{Introduction}

The incidence of esophageal cancer continues to rise in the United States, despite increased efforts for surveillance in Barrett's esophagus [1]. As a result, advances in mucosal imaging have been created to help target neoplasia including high-resolution endoscopy, narrow band imaging (NBI), and confocal laser endomicroscopy. The latest technology that has been developed is called volumetric laser endomicroscopy. This technology captures images up to $3 \mathrm{~mm}$ below the mucosa at a 7-micron resolution in real time. VLE is a second generation of optical coherence tomography that provides real-time high-resolution cross-sectional imaging using a balloon catheter with scanning optics.

With VLE, a 6-cm segment of Barrett's can be imaged in 90 seconds allowing for $100 \%$ of the tissue to be captured. Thus $100 \%$ of the mucosa is optically biopsied. The current gold standard is random biopsies of the Barrett's segment that allows for $<5 \%$ of tissue sampling in long-segment disease $[1,2]$. VLE technology is appealing as it allows a detailed wide field view of the Barrett's mucosa to allow for targeted sampling, thus theoretically maximizing sampling yield while decreasing procedure time. copy diagnosing the Barrett's esophagus. All VLE exams were performed within 6 months of the previous endoscopy. In five patients, VLE-targeted biopsy resulted in upstaged disease/diagnosed dysplasia that then qualified the patient for endoscopic ablation therapy. In one patient, VLE localized a focus of intramucosal cancer that allowed for curative endoscopic mucosal resection. This case series shows that endoscopy with VLE can target neoplasia that cannot be localized by highdefinition endoscopy with NBI and random biopsies.

There are OCT features of normal esophagus, gastric cardia, intestinal metaplasia (IM), IM with dysplasia or cancer that can be seen on VLE [24]. We report six cases of patients with long-segment Barrett's esophagus where endoscopy with VLE was performed within 6 months after a previous standard high-definition endoscopy with narrow-band imaging and biopsies. In all of the cases, VLE was able to diagnose neoplasia (dysplasia or cancer), upstage pathology and target disease.

\section{Patients and methods \\ $\checkmark$}

Six patients with long-segment Barrett's esophagus were selected for this series from the endoscopic database at our institution. Our institution has performed over 200 cases of VLE for Barrett's esophagus, of which roughly half were for longsegment Barrett's esophagus ( $>3 \mathrm{~cm}$ ). Institutional review board approval was obtained for this report. All patients in this series had undergone prior high-definition endoscopy with NBI and random biopsies (every 1 to $2 \mathrm{~cm}$ of the Barrett's in four-quadrant fashion) within 6 months of the endoscopy with VLE that did not reveal any lesions consistent with neoplasia. In these six pa- 


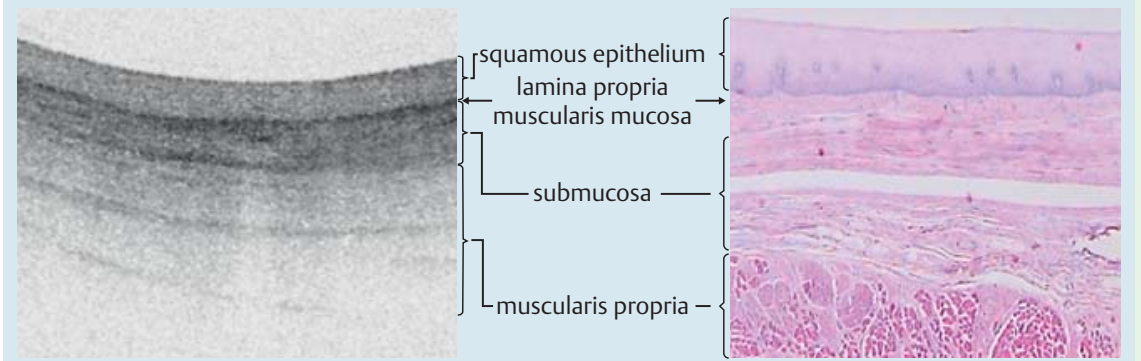

Fig. 1 a VLE image of normal squamous mucosa with the layered architecture without glands. Scale bar is $1 \mathrm{~mm}$ vertical and horizontal. $\mathbf{b}$ Corresponding normal histology.

tients, these were the index endoscopies diagnosing the Barrett's esophagus. All patients were referred for subsequent endoscopy with VLE by their primary gastroenterologist, given that they had long-segment disease; it was felt by the referring gastroenterologist that advanced imaging in a tertiary care setting was better suited for follow-up endoscopy with long-segment disease. The current American College of Gastroenterology guidelines at the time of endoscopy [5] for these patients was to repeat an endoscopy with biopsy within 1 year of diagnosis if no dysplasia was present (4 patents in this series), if low-grade dysplasia was present to repeat an endoscopy within 6 months to exclude high-grade dysplasia (1 patient in this series), and to undergo endoscopic therapy for intramucosal cancer (1 patient in this series). The referring gastroenterologist and patient dictated timing of repeat endoscopy; which was less than 6 months for all cases and thus within a year.

At the time of the VLE all patients included in this series underwent repeat high-definition endoscopy with NBI first, prior to VLE examination. VLE-targeted biopsies were performed on areas suspicious for neoplasia then random biopsies were performed. Patients were not included in this case series if findings on repeat high-definition upper endoscopy, NBI exam, or random biopsies led to a diagnosis of neoplasia. Including these VLE cases in the series would overestimate the potential of VLE to target neoplasia.

All VLE exams were performed using the only commercially available platform in the United States (NvisionVLE ${ }^{\mathrm{TM}}$ Imaging System, NinePoint Medical, Bedford, MA, USA). It is our practice to use a $20-\mathrm{mm}$ balloon catheter unless there is a stricture or tortuous esophagus, in which case, a $14-\mathrm{mm}$ or $17-\mathrm{mm}$ balloon catheter is used. All procedures were performed by endoscopists trained in endoscopy for Barrett's esophagus (AJT, MM). Each has performed over 40 VLE exams prior to the cases included in this series. The endoscopist interpreted the VLE images. A clinical representative from the VLE Company was present to aid the endoscopist in image interpretation, if desired. The pathologists who interpreted the pathology in this study are experts in gastrointestinal pathology and are trained to read Barrett's esophagus histology. All histology with dysplasia is reviewed in a weekly conference among the gastrointestinal pathologists to ensure a high degree of accuracy.

Imaging is performed by automatic helical pullback of the probe from the distal to proximal end of the balloon over a 90-second period to create real-time 360-degree volumetric images. Twelve hundred cross sectional scans are generated over the $6 \mathrm{~cm}$ segment. VLE scans are viewed by using a software interface that allows real time viewing of cross-sectional transverse and longitudinal views. A registration line is seen on the balloon and its orientation is matched up with the registration line on the crosssectional imaging (viewed as a thin shadow line on the VLE im- age) to allow targeting of abnormalities seen. Lesions are localized taking into consideration the lesion's relationship to the GE junction and to the registration line. If multiple scans are required to visualize a long segment of Barrett's esophagus then unique targets are visualized on both scans to overlap them accurately. Once lesions are detected on the VLE scan that are suspicious for neoplasia, the corresponding endoscopic area can be biopsied. Endoscopic mucosal resection can be considered if the abnormal area on the VLE scan is located below the surface. All procedures were performed with monitored anesthesia care without an endotracheal tube. Vital signs and oxygen saturation were monitored and recorded throughout the procedure.

\section{VLE Features of esophageal squamous mucosa,} and intestinal metaplasia

VLE is able to differentiate between normal squamous mucosa and intestinal metaplasia $[2,3,6]$. VLE features of esophageal squamous mucosa include layered horizontal architecture without glands in the epithelium ( Fig. 1). VLE features of intestinal metaplasia include loss of layered architecture in the setting of no surface pits and crypts ( $\bullet$ Fig. 2 a) and glands seen in the epithelium ( Fig. 2b, yellow arrows) [6].

\section{VLE Features of Neoplasia}

There are features of OCT imaging that can help target dysplasia. Glandular architecture and signal intensity are the two main components for determining if dysplasia is present [3,7]. An OCT scoring index (OCT-SI) was created in 2006 to aid in OCT diagnosis of Barrett's esophagus associated dysplasia [3]. A score greater than or equal to 2 is associated with a sensitivity of $83 \%$ and a specificity of $75 \%$ for dysplasia.

Recently Leggett et al devised a new algorithm for detection of dysplasia based on ex vivo endoscopic mucosal resection specimens scanned with VLE. This index is called the VLE diagnostic algorithm (VLE-DA) [7]. The algorithm first determines the degree of mucosal effacement (partial versus full loss of layered mucosal architecture). If complete effacement is seen and the surface intensity is greater than the subsurface, then dysplasia is suspected. If partial effacement is seen with greater than 5 atypical glands, then dysplasia is also suspected. This VLE-DA had a sensitivity of $86 \%$ (95\% CI 69-96) and a specificity of $88 \%$ (95\% $\mathrm{CI}, 60$-99. Finally, a washed-out appearance known as homogeneous scattering is suggestive of high-grade dysplasia or cancer.

\section{Case Reports $\nabla$}

- Table 1 shows the patient characteristics, findings from the first endoscopy, highest histology yield from the first endoscopy, interval between the two exams, findings from the second 
11

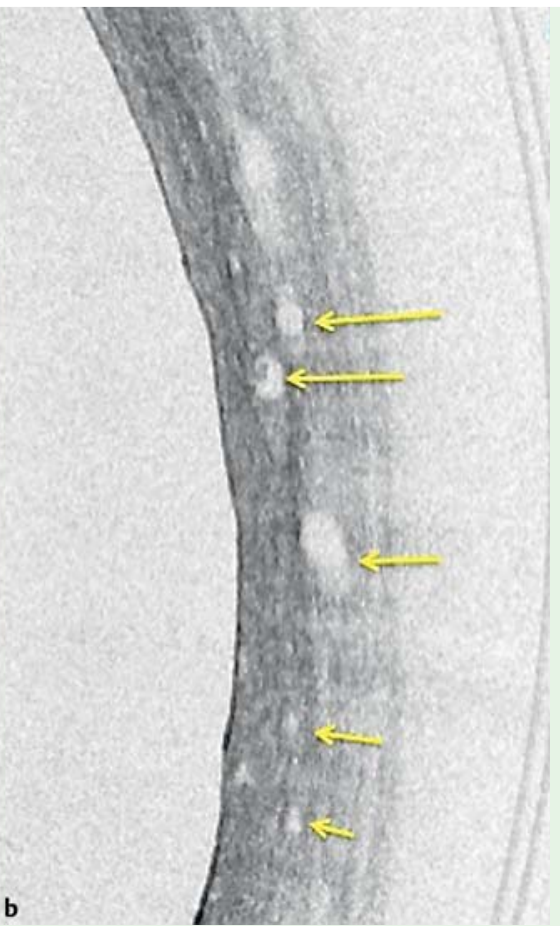

Fig.2 a VLE image showing loss of layered architecture in the setting of no surface pits and crypts. b Atypical glands (yellow arrows) with loss of layered architecture.

endoscopy with VLE, highest histology yield from the VLE exam, and how VLE changed management in all six patients. Fig. 3 shows the corresponding VLE images that were obtained which allowed for targeting of neoplasia.

Patients one through four had all been previously diagnosed with non-dysplastic Barrett's esophagus or classified as indefinite for dysplasia. If they did not undergo VLE, they would have been surveyed with endoscopy. However, they underwent repeat endoscopy with VLE that upstaged their disease to dysplastic Barrett's esophagus. The upstaging of pathology qualified the patients for initiation of ablative therapies for eradication of intestinal metaplasia.

Patient 1 had developed a stricture in his nodular Barrett's segment. Two previous endoscopies were performed with dilation of the stricture and extensive biopsies at the site. The biopsies showed intestinal metaplasia without dysplasia and thus no ablative therapies were offered. After his VLE-targeted biopsy returned as high-grade dysplasia, he was started on cryotherapy after successful stricture dilation, and his disease is improving. Patients 2 through 4 had flat Barrett's mucosa without visible neoplasia and were started on radiofrequency ablation protocols

Table 1 Patient characteristics, findings from the first endoscopy, and subsequent endoscopy with VLE.

\begin{tabular}{|c|c|c|c|c|c|c|}
\hline & Patient 1 & Patient 2 & Patient 3 & Patient 4 & Patient 5 & Patient 6 \\
\hline Age (years) & 82 & 70 & 66 & 77 & 58 & 65 \\
\hline Gender (male/female) & M & $\mathrm{F}$ & M & $\mathrm{F}$ & M & M \\
\hline Length of Barrett's (cm) & 3 & 7 & 9 & 5 & 5 & 4 \\
\hline Hiatal hernia (Yes/No) & Y & Y & Y & Y & $\mathrm{Y}$ & Y \\
\hline Findings on first endoscopy/NBI & $\begin{array}{l}\text { Stricture, } \\
\text { Barrett's } \\
\text { mucosa }\end{array}$ & $\begin{array}{l}\text { Barrett's } \\
\text { mucosa }\end{array}$ & $\begin{array}{l}\text { Barrett's } \\
\text { mucosa }\end{array}$ & $\begin{array}{l}\text { Barrett's } \\
\text { mucosa }\end{array}$ & $\begin{array}{l}\text { Barrett's } \\
\text { mucosa }\end{array}$ & $\begin{array}{l}\text { Barrett's mucosa/no } \\
\text { visible lesion on NBI }\end{array}$ \\
\hline $\begin{array}{l}\text { Highest grade of disease on } \\
\text { histology after } 1^{\text {st }} \text { endoscopy }\end{array}$ & IM & IM & IND & IND & LGD & $\begin{array}{l}\text { IMCA on random } \\
\text { biopsy }\end{array}$ \\
\hline $\begin{array}{l}\text { Time between the first endoscopy } \\
\text { and endoscopy w/ VLE (weeks) }\end{array}$ & 8 & 12 & 4 & 24 & 4 & 1 \\
\hline $\begin{array}{l}\text { Size of VLE balloon catheter used } \\
(\mathrm{mm})\end{array}$ & 14 & 20 & 20 & 20 & 20 & 20 \\
\hline \multirow[t]{3}{*}{ Findings on VLE exam } & ISM & LLA & LLA & LLA & AG & LLA \\
\hline & LLA & ISM & ISM & AG & & AG \\
\hline & IS & & AG & & & Ho \\
\hline $\begin{array}{l}\text { Highest grade of disease on } \\
\text { histology after VLE }\end{array}$ & HGD & LGD & LGD & LGD & HGD & $\begin{array}{l}\text { IMCA-T1a (5-mm } \\
\text { tumor) }\end{array}$ \\
\hline How VLE changed management & $\begin{array}{l}\text { Started } \\
\text { cryotherapy }\end{array}$ & Started RFA & $\begin{array}{l}\text { Started } \\
\text { RFA }\end{array}$ & $\begin{array}{l}\text { Started } \\
\text { RFA }\end{array}$ & Started RFA & $\begin{array}{l}\text { Curative EMR } \\
\text { followed by } \\
\text { cryotherapy }\end{array}$ \\
\hline
\end{tabular}

Abbreviations: NBI, narrow band imaging; IM, intestinal metaplasia; IND, indefinite for dysplasia; LGD, low-grade dysplasia; HGD, high-grade dysplasia; IMCA, intramucosal cancer; ISM, inverted surface maturation; Ho, homogeneous scattering; AG, atypical glands; LLA, loss of layered architecture; IS, irregular surface 


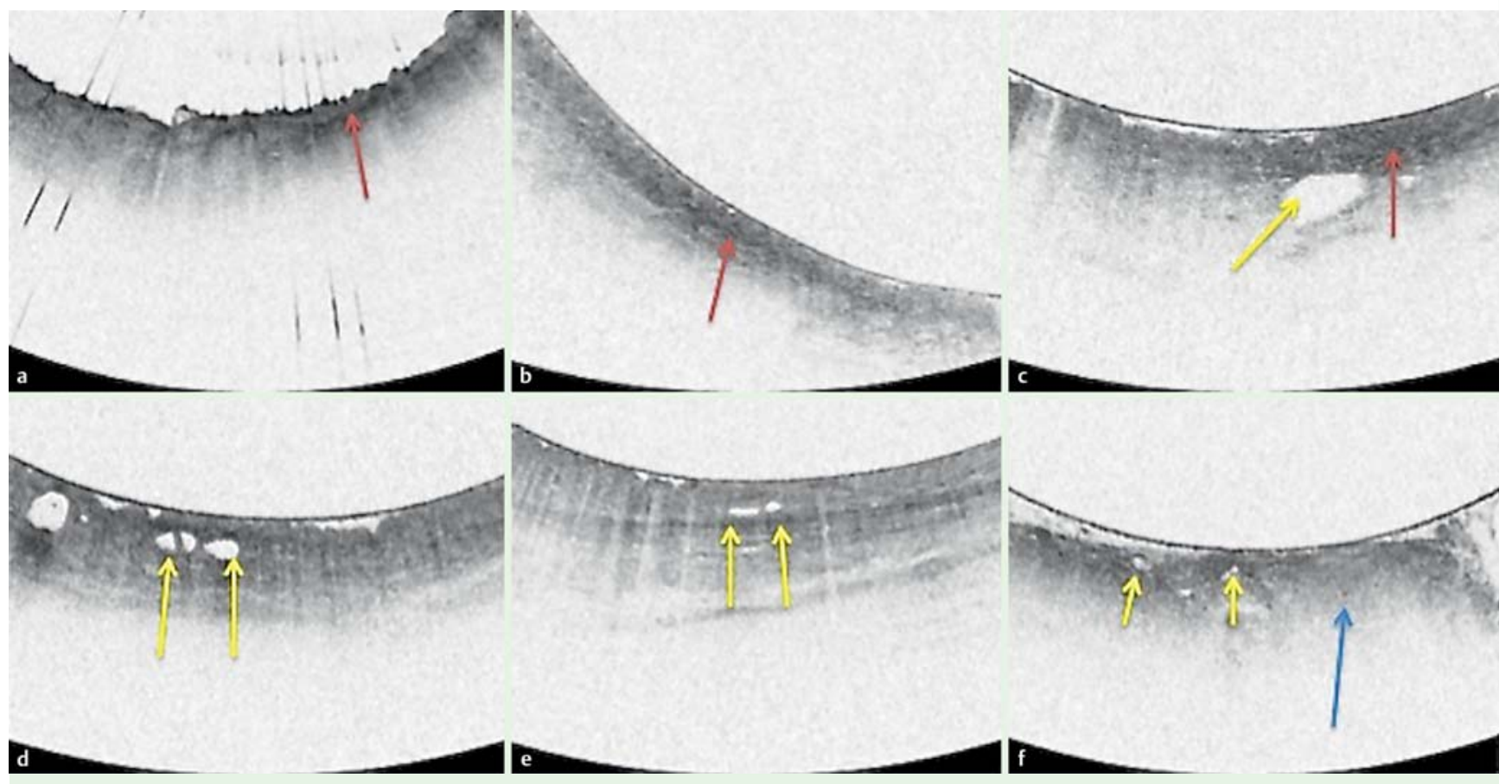

Fig. 3 Volumetric laser endomicroscopy images corresponding to the sites that allowed for targeted biopsies. Patient 1 corresponds to A, Patient 2 to B, etc. Features on VLE that allowed targeting of neoplasia included a surface signal intensity > subsurface intensity (patients 1,2,3; red arrow) and atypical glands (Patients 3,4,5,6; yellow arrow). Patients 1,2,3,4, and 6 had loss of layered architecture consistent with intestinal metaplasia. Patient 6 had homogeneous scattering or a washout appearance suggestive of cancer (blue arrow).

after VLE-targeted biopsies upstaged their disease to dysplastic Barrett's.

Patient 5 had been previously diagnosed with low-grade dysplasia. He was refusing ablative therapy and in favor of surveillance with endoscopy. He opted for VLE at the advice of his primary gastroenterologist. VLE-targeted biopsies revealed HGD that convinced the patient he needed ablation, as the guidelines unequivocally endorse RFA for HGD [5]. Finally Patient 6 had a previous endoscopy consistent with Barrett's esophagus. Random biopsies returned as intramucosal cancer but high-definition white light endoscopy or narrow-band imaging did not show a focal raised lesion to target for endoscopic mucosal resection (EMR). On repeat endoscopy and NBI, a focal lesion could not be identified. VLE targeted a specific area to perform EMR. EMR was performed with adequate margins and histology showed a small 5-mm T1a cancer. VLE allowed for targeted resection of the intramucosal cancer. The patient is undergoing subsequent ablative therapy to eradicate the remaining intestinal metaplasia.

Features on VLE that allowed targeting of neoplasia included a surface signal intensity > subsurface intensity (Patients $1,2,3$ ) and atypical glands (Patients 3-6). Patient 6 had homogeneous scattering or a washout appearance suggestive of cancer. Patients $1,2,3,4$, and 6 has loss of layered architecture consistent with intestinal metaplasia.

No immediate adverse events (AEs) occurred in any of the patients in this case series. Our endoscopy suite makes standard follow-up phone calls within 48 hours of the procedure and documents any pain or delayed AEs events. None of the patients in this series developed any pain or delayed adverse events from the VLE procedure.

\section{Discussion \\ $\nabla$}

VLE is a new imaging technology that allows for real-time targeting of disease for biopsy or endoscopic mucosal resection in Barrett's esophagus. Wolfson et al already reported that VLE is safe and efficacious in Barrett's esophagus in a large number of patients [8] while Sauk et al have shown a high interobserver agreement for interpretation of images among trained readers [2].

This case series demonstrates the potential of VLE to not only target neoplasia, but to target neoplasia that conventional methods could miss. Previous reports have also reported the ability of VLE to detect neoplasia when it is not evident on endoscopy. Leggett et al reported a case of VLE-targeted EMR of subsquamous cancer [4]. Swager et al reported that VLE can target subsquamous glands after radiofrequency ablation; although most of these glands were benign [9]. Our group reported a case, not in this series, of VLE detecting focal HGD in a long segment of Barrett's when two previous endoscopies did not target this [10].

It should be noted that cases series are limited by bias for reporting cases of favorable outcomes. Future studies examining the percentage yield of VLE, sensitivity and specificity of VLE, and cost effectiveness of VLE are needed to determine if VLE will be a sustainable option to screen and survey Barrett's in the future. Some of these questions will be answered from the national VLE registry that is ongoing. As this case series shows, the technology is promising. In summary, this case series shows that VLE can target neoplasia that is not detected by high-definition white light endoscopy, NBI, or random biopsies. This may allow for expeditious ablative therapies to eradicate intestinal metaplasia and prevent progression of disease. Larger studies are needed to confirm our findings.

Competing interests: None 


\section{References}

1 Sharma P, Brill J, Canto $M$ et al. Advanced Imaging in Barrett's Esophagus. Clin Gastroenterol Hepatol 2015

2 SaukJ, Coron E, Kava $L$ et al. Interobserver agreement for the detection of Barrett's esophagus with optical frequency domain imaging. Dig Dis Sci 2013; 58: $2261-2265$

3 Evans JA, Poneros JM, Bouma BE et al. Optical coherence tomography to identify intramucosal carcinoma and high-grade dysplasia in Barrett's esophagus. Clin Gastroenterol Hepatol 2006; 4: 38-43

4 Leggett CL, Gorospe E, Owens VL. Nature Publishing Group. et al. Volumetric laser endomicroscopy detects subsquamous Barrett's adenocarcinoma. Am J Gastroenterol2014; 109: 298 - 299

5 Wang KK, Sampliner RE. Updated Guidelines 2008 for the Diagnosis, Surveillance and Therapy of Barrett's Esophagus. Am J Gastroenterol 2008; 103: $788-797$
6 Poneros JM, Brand S, Bouma BE et al. Diagnosis of specialized intestinal metaplasia by optical coherence tomography. Gastroenterology 2001; 120: $7-12$

7 Leggett CL, Gorospe EC, Chan DK et al. Comparative diagnostic performance of volumetric laser endomicroscopy and confocal laser endomicroscopy in the detection of dysplasia associated with Barrett's esophagus. Gastrointest Endosc 2015

8 Wolfsen HC, Sharma P, Wallace MB et al. Safety and feasibility of volumetric laser endomicroscopy in patients with Barrett's esophagus (with videos). Gastrointest Endosc 2015; 3: 1 - 10

9 Swager A-F, Boerwinkel DF, de Bruin DM et al. Detection of buried Barrett's glands after radiofrequency ablation with volumetric laser endomicroscopy. Gastrointest Endosc 2015: 1 -9

10 Trindade AJ, Vamadevan AS, Sejpal DV. Finding a needle in a haystack: use of volumetric laser endomicroscopy in targeting focal dysplasia in long-segment Barrett's esophagus. Gastrointest Endosc 2015; 82: $756-757$ 